\title{
BOUNDING CURVES IN ALGEBRAIC SURFACES BY GENUS AND CHERN NUMBERS
}

\author{
Steven Shin-Yi Lu And Yoichi MiYaokA
}

\begin{abstract}
This note will provide some effective bounds for the degree of a curve in an algebraic surface of general type with only constraints on the number of ordinary nodes and ordinary triple points of the curve. The degree is taken with respect to the canonical polarization of the surface and the bounds are given linearly in terms of the genus of the curve and the Chern numbers of the surface.
\end{abstract}

\section{Introduction and statement of result}

The work of Bogomolov $[1,2]$ has been an important source of information in the study of the geometry of surfaces. Aside from giving a precise description of each pluricanonical system in the work of I. Reider [10], it provides important cohomological constraints in the form of vanishing theorems for tensorial bundles and Chern number inequalities for such surfaces. The latter has been refined by the works of Miyaoka [7, 9], Yau [12,13] and R. Kobayashi [4]. This paper will focus on the problem of bounding curves in surfaces of general type by their geometric genus, also pioneered by Bogomolov in his works above. Bogomolov's technique relies on the brilliant idea of lifting curves to the tangent bundle of the surface but uses foliation arguments that are difficult to make effective. His results include bounding the family of smooth curves and curves of negative selfintersection by their geometric genus in any surface of general type and bounding arbitrary curves by their geometric genus in a surface of general type with $c_{1}^{2}>c_{2}$.

In this paper, we obtain effective versions of these results of Bogomolov with a view towards resolving a conjecture of Serge Lang in a simple case, that of the finiteness of rational curves and elliptic curves in a surface of general type. We achieve this by exploiting the Miyaoka-Yau inequality for appropriate open algebraic surfaces and also by giving a version of the inequality valid more generally for two dimensional "log-schemes," see theorem 4 for the precise statement. We like to take this opportunity to

Received September 9, 1994; Revised June 23, 1995.

First author supported in part by a JSPS postdoctoral fellowship. 
mention that a more general inequality we established in arbitrary dimension yields directly the following partial resolution of the same conjecture of Lang but in any dimension. Recall first that, on a smooth projective variety, a divisor is called pseudoeffective if its intersection with any curve is nonnegative and a divisor representing the first Chern class of the variety is called the anticanonical divisor.

Theorem 0. [6] Let $X$ be a nonsingular projective variety. If $X$ is of general type, then $X$ has only a finite number of nonsingular codimension-one subvarieties having pseudo-effective anticanonical divisor. In particular, $X$ has only a finite number of nonsingular codimension-one Fano, Abelian, and Calabi-Yau subvarieties.

One of the best procedures we use to get the most out of the MiyaokaYau inequality involves a resolution of curve singularities analogous to logminimal resolution of open surfaces singularities. In this way we are able to give effective bounds on the degree with respect to the canonical polarization of the surface for curves of bounded arithmetic genus, for curves of bounded topological Euler characteristic, for curves of bounded geometric genus and self-intersection from above, and for curves of bounded geometric genus having an upper bound on the number of ordinary nodes and ordinary triple points less the number of cusps (counted by order) and higher multiplicity singularities. We also bound effectively normal crossing curves of bounded geometric genus in a surface of general type with $c_{1}^{2}>c_{2}$. It will follow therefore that curves of the above types are bounded and that those with geometric genus zero or one are finite in number in a surface of general type. We can also bound effectively curves of a fixed genus in a surface of general type whose singularities consist entirely of ordinary nodes or entirely without ordinary nodes, but the procedures are completely different and will thus be given elsewhere.

We first show how one can obtain the following effective estimates by applying the Miyaoka-Yau inequality for open algebraic surfaces as given by Y. Miyaoka in [9], see also F. Sakai [11]. We note that the same inequality for slightly less general open surfaces was given by Yau in $[15,3]$, by Tian and Yau in [14] and by R. Kobayashi in [4]. We will identify a line bundle with a divisor to which it corresponds and write intersections of divisors multiplicatively.

Theorem 1. Let $C$ be an irreducible curve of geometric genus $g$ in a surface $S$. Let $K=K_{S}$ be the canonical bundle and $c_{1}, c_{2}$ be the Chern classes of $S$. Let $\Delta=3 c_{2}-c_{1}^{2}$. If some multiple of $K+C$ is effective, then the following inequalities hold: 


$$
\begin{aligned}
C^{2} & \geq-\Delta-2 g+2+n_{\text {ord }} \\
K C & \leq \Delta+2 g-2-\chi_{\text {top }}(C) \\
K C & \leq 2 \Delta+6 g-6+C^{2} \\
K C & \leq \Delta+4 g-4+n_{\text {ord }},
\end{aligned}
$$

where $n_{\text {ord }}$ is the number of ordinary nodes and ordinary triple points of $C$ and $\chi_{\text {top }}(C)$ is the topological Euler Characteristic of $C$.

Remark. It will be given in the proof that each infinitely near singularity of $C$ (including those of $C$ ) which is not an ordinary node gives an additional, positive, and good contribution to each of the inequalities above, see also the remark in Section 3. But we choose not to display them in the above theorem.

We will prove this theorem first for the case that $C$ is a normal crossing to motivate the procedure. We then lay out a setup which takes care of (1)-(3) giving also the contributions from the singularities, see lemma 1. Finally we refine the setup in two ways to obtain (4) twice.

Let $p_{a}=(1 / 2)(K+C) C+1$, the arithmetic genus of $C$. One can verify the following inequalities by resolving the singularities of $C$ and the adjunction formula:

$$
2 g-2 \leq-\chi_{\text {top }}(C) \leq 2 p_{a}-2 .
$$

Observe then that the canonical degree of $C, K C$, has the following bounds:

(a) By (2) above and the adjunction formula

$$
\begin{aligned}
K C & \leq \Delta-2 \chi_{t o p}(C) \\
& \leq \Delta+4 p_{a}-4 .
\end{aligned}
$$

(b) If $C^{2} \leq 0$, then by (3),

$$
K C \leq 2 \Delta+6 g-6 .
$$

(c) If $C$ is smooth, then we get the better estimate

$$
K C \leq \Delta+4 g-4
$$

by (4) or by (a) above. 
We thus recover Bogomolov's results of boundedness of curves of nonpositive self-intersection and of curves which are nonsingular in a surface of general type but with effective uniform estimates, constant in any family of surfaces. We now quote the classic result of Bogomolov from which he derived these boundedness results.

Theorem 2. (Bogomolov) If $S$ is a surface of general type with $c_{1}^{2}(S)>$ $c_{2}(S)$, then curves in $S$ are bounded by their geometric genus, i.e., curves of a fixed geometric genus lie in a bounded family.

Remark. Given a surface of general type with $c_{1}^{2}>c_{2}$, one can deduce from Bogomolov's argument that there is a constant $A$ depending on $S$ such that

$$
K C<\frac{6 c_{2}}{c_{1}^{2}-c_{2}}(g-1)+A
$$

for any curve $C$ with geometric genus $g$. Unfortunately, it is not possible to estimate $A$ directly by his technique.

An effective version of this result will be derived via the Miyaoka-Yau inequality for "log-surfaces" with fractional boundary divisors and is as follows:

Theorem 3. Let $S$ be a surface of general type with $c_{1}^{2}(S)>c_{2}(S)$. Let $K$ be the canonical bundle of $S$ and let $C$ be a curve of geometric genus $g$ with only ordinary nodal singularities in $S$. Then we have

$$
K C \leq \frac{3 \Delta+4 g-4}{c_{1}^{2}(S)-c_{2}(S)} K^{2},
$$

where the factor 3 can be dropped in the case $g>0$.

This result was given by G. Tian using Kähler-Einstein metrics in the C.I.M.E. lectures, in the case $K$ is nef. Our version here is slightly more general in that we do not need the canonical bundle to be nef (nor the positivity of the self-intersection of the curve) in order to have the necessary Chern number inequality (c.f., our Theorem 4), but otherwise we follow the proof given by Tian in his C.I.M.E. lectures.

As a corollary of theorem 1, we obtain a partial resolution of the aforementioned conjecture of Lang [5], with constraint on two types of singularities of the curves but without any constraint on the surface of general type.

Corollary 1. In a surface of general type, there are only a finite number of rational curves and elliptic curves with a fixed bound on the number of ordinary nodes and ordinary triple points of each curve. 
We reiterate that curves of a given genus with only ordinary nodes or without ordinary nodes can be bounded by different techniques. Implications in hyperbolic geometry, and applications to the (effective) MordellLang conjectures over function fields, are relegated elsewhere.

\section{Proof of Theorem 1}

\section{Nodal Case:}

We first assume $C$ has $n$ ordinary nodes and no other singularities. The Miyaoka-Yau inequality for the locally free sheaf $V=\Omega_{S}(\log C)$ is valid, [11]. So

$$
(K+C)^{2}=c_{1}^{2}(V) \leq 3 c_{2}(V)=3 e(S \backslash C)=3(e(S)-e(C)),
$$

where $e(S), e(C)$ are the topological Euler characteristics of $S$ and $C$, respectively. Putting $-2 P=(K+C) C=2 g-2+2 n$ and noting that $e(S)=c_{2}$ and $e(C)=2-2 g-n$ in our setup, we find

$$
K^{2}-C^{2}-4 P \leq 3\left(c_{2}+2 g-2+n\right)
$$

Hence

$$
C^{2} \geq c_{1}^{2}-3 c_{2}-2 g+2+n,
$$

yielding (1). Similarly, we find

$$
(K+C)^{2}+3\left(K^{2}-C^{2}-4 P\right)=4 c_{1}^{2}(V) \leq 12 c_{2}(V)
$$

and

$$
(K+C)^{2}=(K+C) K-2 P=c_{1}^{2}(V) \leq 3 c_{2}(V),
$$

which give, respectively,

$$
K C \leq 2 \Delta+6 g-6+C^{2} \text { and } K C \leq \Delta+4 g-4+n .
$$

These yield (3) and (4) (and hence (2)) of theorem 1, respectively.

\section{General Case:}

With the setup as in Theorem 1, we consider a sequence of blowups $\sigma_{i}: S_{i} \rightarrow S_{i-1}$ and define a curve $C_{i}$ in $S_{i}$ inductively as follows. Let $C_{0}=C$ and $S_{0}=S$. Let $p_{i}$ be a singular point of $C_{i-1}$ with multiplicity $m_{i}$. We always assume that $p_{i}$ is not an ordinary node of $C_{i-1}$. Let $\sigma_{i}$ be the blowup of $S_{i-1}$ with center $p_{i}$. We define $C_{i}$ to be the strict transform of $C_{i-1}$ if $m_{i}>2$ and to be its reduced total transform otherwise. Note that $C_{i}$ need not be irreducible here. This process ends after a finite 
number of steps at a normal crossing curve, $\hat{C}=C_{N}$ in $\hat{S}=S_{N}$ say. The picture is

$$
\begin{aligned}
& \hat{S}=S_{N} \stackrel{\sigma_{N}}{\longrightarrow} S_{N-1} \stackrel{\sigma_{N_{1}}}{\longrightarrow} S_{N-2} \ldots \quad S_{1} \stackrel{\sigma_{1}}{\longrightarrow} S_{0}=S \\
& \cup \cup \cup \cup \cup \cup \cup \\
& \hat{C}=C_{N} \longrightarrow C_{N-1} \longrightarrow C_{N-2} \ldots C_{1} \longrightarrow C_{0}=C \text {. }
\end{aligned}
$$

We may write $\hat{C}=\bar{C}+F$ where $\bar{C}$ is the strict transform of $C$ from $S$ and $F$ is the exceptional divisor. We observe that the nodes of $\bar{C}$ form a subset of the infinitely near nodes of $C{ }^{1}$ So if their numbers are $n^{\prime}$ and $n$, respectively, we have $n^{\prime} \leq n$. We call these $n^{\prime}$ nodes odd since the number of infinitely near double points of $C$ over which one of these nodes sits is odd. Similarly, we define odd simple infinitely near cusps of $C$ and let $c^{\prime}$ be their number. Let $l$ be the number of infinitely near double points of $C$ which are not simple nodes. (1)-(3) of Theorem 1 is a corollary of the following computational result.

Lemma 1. If a multiple of $K+C$ is effective, then

$$
\begin{aligned}
& \text { (1) } C^{2} \geq-\Delta-2 g+2+n+2 l+\sum^{\prime \prime}\left(n_{i}^{2}-4\right) \\
& \text { (2) } K C \leq 2 \Delta+6 g-6+C^{2}-2 l-\sum^{\prime \prime}\left(n_{i}^{2}+n-8\right) \\
& \text { (3) } K C \leq \Delta+4 g-4+n^{\prime}-c^{\prime}+\sum^{\prime \prime}\left(4-n_{i}\right) \text {, }
\end{aligned}
$$

where $\sum^{\prime \prime}$ means summing over infinitely near points of $C$ with multiplicity $n_{i}>2$.

Proof. As we do not blow up nodes, $F$ is a disjoint union of $s$ rational curves, each coming from a $\left(m_{i}=2\right)$-centered blowup and each intersecting $\bar{C}$ at no more than one point. Let $d$ be the number of rational curves in $F$ disjoint from $\bar{C}$. We see that $\hat{C}$ has topological Euler characteristic $\hat{e}=\left(-2 g+2-n^{\prime}\right)+s+d$. Let $\hat{K}$ be the canonical bundle of $\hat{X}$. Let $\hat{P}$ be the Euler characteristic of the sheaf $\mathcal{O}_{\hat{C}}$. The adjunction formula tells us that

$$
-2 \hat{P}=(\hat{K}+\hat{C}) \hat{C}=\left(2 g-2+2 n^{\prime}\right)-2 d
$$

We also have

$$
\begin{aligned}
\hat{K} & =K+\sum_{i} E_{i} \\
\hat{K}+\hat{C} & =K+C-\sum^{\prime}\left(m_{i}-1\right) E_{i},
\end{aligned}
$$

\footnotetext{
${ }^{1}$ By an infinitely near singularity here, we mean a singularity of $C$ along with those of the strict transform of $C$ on the exceptional divisor of some blowup in the sequence of blowups defined above.
} 
where $E_{i}$ is the total transform of the exceptional divisor of $\sigma_{i}$ and where $\sum^{\prime}$ means summing over the $m_{i}>2$ points. Let $\hat{c}_{i}$ be the Chern classes of the logarithmic cotangent sheaf $\Omega_{\hat{S}}(\log \hat{C})$. We may write

$$
\begin{aligned}
& \hat{c}_{1}^{2}=\hat{K}^{2}-\hat{C}^{2}-4 \hat{P}=K^{2}-C^{2}+\sum^{\prime}\left(m_{i}^{2}-1\right)+4(g-1+n-d) \\
& \hat{c}_{2}=c_{2}(\hat{S})-\hat{e}=c_{2}+N-\hat{e}=c_{2}+2 g-2+r+n^{\prime}-d,
\end{aligned}
$$

where $r=N-s$ is the number of blowups with $m_{i}>2$. The hypothesis of our lemma implies that a multiple of $\hat{K}+\hat{C}$ is effective and so $\hat{c}_{1}^{2} \leq 3 \hat{c}_{2}$, cf. [9]. Hence

$$
C^{2} \geq c_{1}^{2}-3 c_{2}-2 g+2+n^{\prime}-d+\sum^{\prime}\left(m_{i}^{2}-4\right),
$$

yielding (1) after some case-checking.

Similarly, writing $4 \hat{c}_{1}^{2}$ as $(K+C)^{2}-\sum^{\prime}\left(m_{i}-1\right)^{2}+3\left(\hat{K}^{2}-\hat{C}^{2}-4 \hat{P}\right)$ and writing $\hat{c}_{1}^{2}$ as $(K+C) K+\sum^{\prime}\left(m_{i}-1\right)-2 \hat{P}$, we obtain

$$
\begin{aligned}
& K C \leq 2 \Delta+6 g-6+C^{2}-\sum^{\prime}\left(m_{i}^{2}+m_{i}-8\right) \quad \text { and } \\
& K C \leq \Delta+4 g-4+n^{\prime}-d+\sum^{\prime}\left(4-m_{i}\right),
\end{aligned}
$$

yielding (3) and (2), respectively and completing the proof of Lemma 1.

Remark. Had we used $\hat{c}_{1}^{2} \leq 2 \hat{c}_{2}$, which is the case if $\Omega_{\hat{X}}(\log \hat{C})$ is not almost everywhere ample by a lemma of Miyaoka [8], we would have obtained

$$
\begin{array}{llll}
\text { (a) } C^{2} \geq c_{1}^{2}-2 c_{2} & +2 n+2 l & +\sum^{\prime \prime}\left(n_{i}^{2}-3\right) \\
\text { (b) } K C \leq & \quad K c_{2}-c_{1}^{2} & +2 g-2 & -\sum^{\prime \prime}\left(n_{i}-3\right) .
\end{array}
$$

Note in particular that the lower bound for $C^{2}$ is independent of $g$ in this case.

In order to obtain (4) of theorem 1 , we redefine our sequence of blowup transforms as follows:

$$
\begin{aligned}
& \hat{S}=S_{N} \stackrel{\sigma_{N}}{\longrightarrow} S_{N-1} \stackrel{\sigma_{N_{1}}}{\longrightarrow} S_{N-2} \ldots \quad S_{1} \stackrel{\sigma_{1}}{\longrightarrow} S_{0}=S \\
& \cup \cup \cup \cup \cup \cup \cup \\
& \hat{C}=C_{N} \longrightarrow C_{N-1} \longrightarrow C_{N-2} \ldots C_{1} \longrightarrow C_{0}=C \text {, }
\end{aligned}
$$

where $\sigma_{i}$ is the blowup of $S_{i-1}$ centered at a singular point $p_{i}$ of $C_{i-1}$ with multiplicity $m_{i}$. We always assume $p_{i}$ is not an ordinary node of $C_{i-1}$. We call it type B if it is an ordinary triple point of $C_{i-1}$ or if $m_{i}>3$, and type A otherwise. We define $C_{i}$ inductively to be the strict transform of $C_{i-1}$ via $\sigma_{i}$ if it is type $\mathrm{B}$, and to be its reduced total transform otherwise. Again this process ends after a finite number of steps at a normal crossing curve $\hat{C}=C_{N}$ in $\hat{S}=S_{N}$. We may write $\hat{C}=\bar{C}+F$ and $\hat{C}_{i}=\bar{C}_{i}+F_{i}$ where $\bar{C}$ and $\bar{C}_{i}$ are the strict transforms of $C$ from $S$ and where $F$ and $F_{i}$ consist of chains of rational curves. Let $E_{i}$ be the exceptional divisor of $\sigma_{i}$. 
Claim(i): Each connected component of $F_{i}$ intersects $\bar{C}_{i}$ at no more than two points with total intersection multiplicity at most three. And if it does intersect at two points, then one of them is an ordinary node of $C_{i}$ while $F_{i}$ and $\bar{C}_{i}$ intersect with multiplicity two at the other.

Proof. Claim(0) is true. Assume $\operatorname{claim}(i-1)$ is true. If $p_{i} \in C_{i-1}$ is type $\mathrm{B}$, then clearly claim $(i)$ is true. So let $p_{i}$ be type A. We consider three cases separately.

Case $p_{i} \notin F_{i-1}$ : Then $\bar{C}_{i}$ must intersect $E_{i}$ at no more than two points with total intersection multiplicity $m_{i}$. If $m_{i}=2$, then only one intersection point can occur as we do not blow up nodes. If $m_{i}=3$ and two intersection points occur, then $E_{i}$ intersects $\bar{C}_{i}$ with multiplicity two at one point and multiplicity one at the other point. Hence the latter point must be an ordinary node and $\operatorname{claim}(i)$ is true in this case.

Case $F_{i-1}$ intersects $\bar{C}_{i-1}$ with multiplicity three at $p_{i}$ : Then they do not intersect elsewhere by the induction hypothesis and $C_{i-1}$ has multiplicity three at $p_{i}$ as we assumed it to be type A. This is only possible if $F_{i-1}$ intersects nontransversally at an ordinary node of $\bar{C}_{i-1}$. Hence $F_{i-1}$ must be the exceptional curve of a previous blowup, say $\sigma_{j}$, and $C_{j-1}$ consists locally at $p_{j}$ of an ordinary cusp component and a smooth component of $\bar{C}_{i-2}$ intersecting nontransversally, i.e., with multiplicity three. Hence $F_{i}$ consists of two smooth rational curves intersecting transversally at two smooth points of $\bar{C}_{i}$, one at a smooth point of $F_{i}$ and the other at the singular point of $F_{i}$. So claim $(i)$ is true in this case.

Case $F_{i-1}$ intersects $\bar{C}_{i-1}$ with multiplicity two at $p_{i}$ : As $p_{i}$ is not an ordinary triple point, $E_{i}$ can only intersect $\bar{C}_{i}$ at one point $p$. If $\bar{C}_{i}$ is smooth at $p$, then either $p$ is a tacnode of $C_{i}$ resulting from blowing up a cusp of $\bar{C}_{i-1}$ or $p$ is an ordinary triple point of $C_{i}$ resulting from blowing up a tacnode of $C_{i-1}$. Otherwise $p$ is outside the strict transform of $F_{i-1}$. In all cases, $\operatorname{claim}(i)$ follows.

It follows that each connected component of $F$ intersects $\bar{C}$ at no more than one point. Let $d$ be the number of components disjoint from $\bar{C}$. Let $\hat{c}_{i}$ be the Chern classes of $\Omega_{\hat{X}}(\log \hat{C})$ as before. An easy computation gives

$$
\hat{c}_{2}=c_{2}+n_{B}+2 g-2+n^{\prime}-d,
$$

where $n_{B}$ is the number of type $B$ blowups and $n^{\prime}$ is the number of nodes of $\bar{C}$. Now $\hat{c}_{1}=\hat{K}+\hat{C}$. The adjunction formula says

$$
-2 \hat{P}=(\hat{K}+\hat{C}) \hat{C}=\left(2 g-2+2 n^{\prime}\right)-2 d .
$$

We use the same symbols $K$ and $C$ for the pull back of $K$ and $C$ to $\hat{S}$, respectively. Let $E_{i}$ be the divisor in $\hat{S}$ defined by the total transforms of the exceptional divisor of $\sigma_{i}$. We divide the index set of $i$ into four sets, $D$, 
$J, T$, and $G$ by the type of singularity of $C_{i-1}$ at $p_{i}$, where $D$ corresponds to double points, $J$ to non-ordinary triple points, $T$ to ordinary triple points, and $G$ to the $m_{i} \geq 4$ points. We have

$$
\begin{aligned}
\hat{K} & =K+\sum_{i \in D} E_{i}+\sum_{i \in J} E_{i}+\sum_{i \in T} E_{i}+\sum_{i \in G} E_{i} \\
\hat{C} & =C-\sum_{i \in D} E_{i}-2 \sum_{i \in J} E_{i}-3 \sum_{i \in T} E_{i}-\sum_{i \in G} m_{i} E_{i} \\
\hat{K}+\hat{C} & =K+C-\sum_{i \in J} E_{i}-2 \sum_{i \in T} E_{i}-\sum_{i \in G}\left(m_{i}-1\right) E_{i} .
\end{aligned}
$$

Hence

$$
(\hat{K}+\hat{C}) \hat{K}=(K+C) K+n_{J}+2 n_{T}+\sum_{i \in G}\left(m_{i}-1\right),
$$

where $n_{J}$ and $n_{T}$ are the number of elements in $J$ and $T$, respectively. Substituting these into $(\hat{K}+\hat{C}) \hat{K}-2 P=\hat{c}_{1}^{2} \leq 3 \hat{c}_{2}$ yields

$$
K C \leq \Delta+4 g-4+n_{T}-n_{J}+n^{\prime}-d-\sum_{i \in G}\left(m_{i}-4\right) .
$$

Now, there are only three type of ordinary triple point singularities in our process. One of them is an infinitely near triple point singularity of $C$ and a simple case checking on the other two shows that

$$
n_{T}-d \leq n_{T}^{\prime}-d_{B}-c^{\prime}-d^{\prime \prime}
$$

where $n_{T}^{\prime}$ is the number of infinitely near ordinary triple points of $C, d_{B}$ is the number of rational curves disconnected from the strict transform of $C$ from type B blowups, $c^{\prime}$ is the number of infinitely near cusps of $C$ of any order and $d^{\prime \prime}$ is the number of infinitely near tacnodes of $C$ which are not ordinary (i.e., having order of contact greater than one). Clearly $n_{J}+d_{B} \geq n_{J}^{\prime}$, where $n_{J}^{\prime}$ is the number of infinitely near nonordinary triple points of $C$. We now partition $G$ into $G^{0}$ and $G^{\prime \prime}$, where $G^{0}$ corresponds to the singularities of $C$ while $G^{\prime \prime}$ corresponds to the rest. Setting $R=$ $c^{\prime}+d^{\prime \prime}+n_{J}^{\prime}+\sum_{i \in G^{\prime \prime}}\left(m_{i}-4\right)$, we have

$$
K C \leq \Delta+4 g-4+n_{T}^{\prime}+n^{\prime}-\sum_{i \in G^{0}}\left(m_{i}-4\right)-R .
$$

Let $n_{i}^{\prime}$ be the number of infinitely near ordinary double points of $C$ and ordinary triple points of $C$ setting above a singularity of $C$ of multiplicity 
$m_{i} \geq 4$ for $i \in G^{0}$. We may write

$$
n_{T}^{\prime}+n^{\prime}-\sum_{i \in G^{0}}\left(m_{i}-4\right)=n_{\text {ord }}+\sum_{i \in G^{0}}\left[n_{i}^{\prime}-\left(m_{i}-4\right)\right] .
$$

Dividing $G^{0}$ into $G_{0}$ and $G_{0}^{\prime}$ where the former consists of those $i$ in $G^{0}$ such that $n_{i}^{\prime}>m_{i}-4$, we may set $R^{\prime}=R+\sum_{i \in G_{0}^{\prime}}\left(n_{i}^{\prime}-m_{i}+4\right) \geq 0$ and write

$$
K C \leq \Delta+4 g-4+n_{\text {ord }}+S_{m_{i} \leq 6}-R^{\prime},
$$

where $S_{m_{i} \leq 6}=\sum_{i \in G_{0}}\left(n_{i}^{\prime}-m_{i}+4\right)$ is a sum over certain $m_{i} \leq 6$ singularities of $C$ (since $n_{i}^{\prime} \leq m_{i} / 2$ by construction so that $n_{i}^{\prime} \leq m_{i}-4$ for $m_{i} \geq 7$ ). We can now redefine our procedure to take care of these $m_{i} \leq 6$ singularities of $C$ in $G_{0}$. One possibility is to take the reduced total transform for every blowup above these points. We claim that this new procedure eliminates their bad contributions to the upper bound for $K C$, so that

$$
K C \leq \Delta+4 g-4+n_{\text {ord }}-R^{\prime}
$$

This will establish the theorem.

This claim will follow easily from the following lemma, which will also offer an alternative proof of the theorem but without the good contributions from the higher order singularities as given above.

Lemma 2. With the setup as in the theorem, we consider a resolution of singularities of $C$ where the reduced total transform of $C$ is a normal crossing divisor $\hat{C}$ in $\hat{S}$. Let $C_{i-1}$ be the reduced total transform of $C$ at the $(i-1)$-st stage with a singularity $p_{i}$ of multiplicity $m_{i}$ defining the $i$-th stage. Let $C_{i}=\bar{C}_{i}+F_{i}$ where $\bar{C}_{i}$ is the strict transform of $C$ as before. Then

$$
K C \leq \Delta+4 g-4+n-\sum_{p_{i} \in F_{i-1}}\left(m_{i}-2\right),
$$

where $n$ is the number of singularities of $C$.

Remark. The last sum gives a nontrivial contribution for every singularity which is not ordinary, i.e., whose total transform via one blowup is not a normal crossing. Hence, (4) of theorem 1 would follow directly from this lemma if the procedure as given in the lemma is changed so that the strict transform rather than the reduced total transform is taken for ordinary singularities of $C$ of multiplicity greater than three. 
Proof of Lemma. With $E_{i}$ as the total transform of the exceptional divisor of the $i$-th blowup, we have

So

$$
\begin{gathered}
\hat{C}=C-\sum\left(m_{i}-1\right) E_{i} \\
\hat{K}=K+\sum E_{i} .
\end{gathered}
$$

$$
(\hat{K}+\hat{C}) \hat{K}=(K+C) K+\sum\left(m_{i}-2\right) .
$$

Let $\hat{C}=\bar{C}+F$ where $\bar{C}$ is the strict transform as before. For $p \in C$, let $n_{p}$ be the number of points in the preimage of $p$ in $\bar{C}$ and let $F_{p}$ be the preimage of $p$ in $F$. As $F_{p}$ is a tree of rational curves, it has arithmetic genus zero. Thus the adjunction formula gives

$$
(\hat{K}+\hat{C}) \hat{C}=2 g-2+2 \sum_{p \in C}\left(n_{p}-1\right) .
$$

Also the topological Euler number of $C$ is

$$
e(C)=2-2 g-\sum_{p \in C}\left(n_{p}-1\right) .
$$

Substituting these into the inequality $(\hat{K}+\hat{C})^{2} \leq 3 \hat{c}_{2}=3\left(c_{2}-e(C)\right)$ yields

$$
K C \leq \Delta+4 g-4+\sum_{p \in C}\left(n_{p}-1\right)-\sum\left(m_{i}-2\right) .
$$

The lemma now follows by noting that $n_{p}$ cannot be greater than $m_{i}$ for the first $p_{i}$ that is in the preimage of $p$.

\section{Log-schemes and other refinements}

Let $D$ be a normal crossing divisor in a surface $X$. We will use the fact, established in Miyaoka [9], that any locally free subsheaf of $\Omega_{S}(\log D)$ whose determinant sheaf is effective up to a multiple satisfies the Chern number inequality $c_{1}^{2} \leq 3 c_{2}$. Let $C$ be a reduced curve in a surface $S$ with only nodes as singularities. Let $C_{1}, C_{2}, \ldots, C_{n}$ be the irreducible components of $C$. Let $\alpha=\left(a_{1}, a_{2}, \ldots, a_{n}\right)$ be a $n$-tuple of positive rational numbers between 0 and 1 , inclusive and let $C_{\alpha}$ be the $Q$-divisor $\sum a_{i} C_{i}$. Then there exist a generically finite cover $S^{\prime}$ of $S$ such that the pull back $C^{\prime}$ of $C$ is an integral divisor whose reduction has only ordinary nodal singularities and such that a locally free subsheaf $\mathcal{E}_{\alpha}$ of $\Omega_{S^{\prime}}\left(\log C_{\text {red }}^{\prime}\right)$ exists whose localization outside $C^{\prime}$ is the same as that of the pull back of $\Omega_{S}$ and whose determinant is $K_{S}+C_{\alpha}$. The construction is easily and best understood in terms of "log-schemes" as originally put forth by Deligne, Mumford, et al, and will be explained in another paper. A simple 
computation of Chern numbers coupled with the above inequality for this subsheaf gives

Theorem 4. Let $C$ be a reduced curve in a surface $S$ of general type with irreducible components $C_{1}, C_{2}, \ldots, C_{n}$. Let $\alpha=\left(a_{1}, \ldots, a_{n}\right)$ be an $n$-tuple of rational numbers such that $0 \leq a_{i} \leq 1$, and $C_{\alpha}=\sum a_{i} C_{i}$. If a multiple of $K_{S}+C_{\alpha}$ is effective, then

$$
\left(K_{S}+C_{\alpha}\right)^{2} \leq 3\left(c_{2}(S)+\sum a_{i}\left(K_{S}+C_{i}\right) C_{i}+\sum_{i<j} a_{i} a_{j} C_{i} C_{j}-\sum a_{i}\left(2-a_{i}\right) n_{i}\right),
$$

where $n_{i}$ is the number of nodes of $C_{i}$.

Remark. One application of this theorem is that we can improve (4) of theorem 1 by reducing the contribution of each ordinary triple point from 1 to $3 / 4$. This is accomplished by taking one half of each exceptional divisor of the blowup of ordinary triple points and applying the theorem above to this fractional log-complex. It is left as an easy exercise.

Proof of theorem 3. Since $C$ has only ordinary nodal singularities and $K$ is effective up to a multiple, we have for $0 \leq a \leq 1$ that

$$
(K+a C)^{2} \leq 3\left(c_{2}+a(K+C) C-a(2-a) n\right),
$$

where $n$ is the number of nodes of $C$. By the adjunction formula

$$
(K+C) C=2 g-2+2 n .
$$

Eliminating $n$ gives

$$
6 a(2-a)(g-1)+a(4-3 a) K C-a^{2} C^{2} \leq 2 \Delta,
$$

or

$$
-\left(3 K C+C^{2}+6-6 g\right) a^{2}+2(2 K C+6-6 g) a \leq 2 \Delta .
$$

We may assume that $3 K C+C^{2}>6 g-6$ as otherwise $K C \leq 2 g-2$. Then the above inequality is optimized when its left-hand side reaches the maximum at

$$
a=\frac{(2 K C+6 g-6)^{2}}{3 K C+C^{2}+6-6 g},
$$

giving

$$
(2 K C+6-6 g)^{2} \leq 2 \Delta\left(3 K C+C^{2}+6-6 g\right) .
$$

The index theorem $K^{2} C^{2} \leq(K C)^{2}$ allows us to eliminate $C^{2}$ in the above inequality, thus obtaining a quadratic in $K C$ :

$2\left(K^{2}-c_{2}\right)(K C)^{2}-(2 \Delta+8 g-8) K^{2}(K C)+(2 \Delta+6 g-6)(2 g-2) K^{2} \Delta \leq 0$.

Theorem 3 now follows by an easy computation. 


\section{Concluding remarks}

In closing, we remark that the same estimates carry over to open algebraic surfaces where the Chern classes are replaced by the logarithmic Chern classes and the geometric genus $g$ is replaced by $1-\chi / 2$, the loggenus, where $\chi=\chi(\tilde{C})$ is the topological Euler Characteristic of the curve properly contained in the open surface. Hence, we see that there are only a finite number of curves of log-genus at most one in an open surface of log-general type where the singularities are as prescribed in this paper.

\section{References}

1. F. A. Bogomolov, Holomorphic tensors and vector bundles on projective varieties, Math. USSR Izv. 13 (1978), 499-555.

2. _ Families of curves on a surface of general type, Soviet Math. Dokl. 18 (1977), 1294-1297.

3. S. Y. Cheng and S-T. Yau, Inequalities between Chern numbers of singular Kähler surfaces and characterization of orbit space of discrete group of $S U(2,1)$, Contemporary Math. 49 (1986), 31-43.

4. R. Kobayashi, Einstein-Kähler V-metrics on open Satake V-surfaces with isolated quotient singularities, Math. Ann. 272 (1985), 385-398.

5. S. Lang, Higher dimensional Diophantine problems, Bulletin of the American Mathematics Society 14 (1986), 159-205.

6. S. Lu and Y. Miyaoka, Bounding codimension one subvarieties and a general inequality between Chern numbers, submitted to the American Journal of Mathematics.

7. Y. Miyaoka, On the Chern numbers of surfaces of general type, Invent. Math. 42 (1977), 225-237.

8. __ Algebraic surfaces with positive indices, Progress in Math. 39 (Birkhauser, Boston, Basel, Stuttgart) (1983), 281-301.

9. __ The maximal number of quotient singularities on surfaces with given numerical invariants, Math. Ann. 268 (1984), 159-171.

10. I. Reider, Vector bundles of rank 2 and linear systems on algebraic surfaces, Ann. of Math. 127 (1988), 309-316.

11. F. Sakai, Semi-stable curves on algebraic surfaces and logarithmic pluri-canonical maps, Math. Ann. 254 (1980), 89-120.

12. S-T. Yau, On Calabi's conjecture and some new results in algebraic geometry, Proc. Nat. Acad. Sci. U.S.A. 74 (1977), 1798-1800.

13. On the Ricci curvature of a compact Kähler manifold and the complex Monge-Ampère equation I, Comm. Pure and Appl. Math. 31 (1978), 339-411. 
14. G. Tian and S-T. Yau, Existence of Kähler-Einstein metrics on complete Kähler manifolds and their applications to algebraic geometry, in "Math. aspects of string theory," World Scientific (1988), 574-672.

15. _ Mètriques de Kähler-Einstein sur les variétés ouvertes, Asterisques 58 (1978), 163-169.

Department of Pure Mathematics, University of Waterloo, Waterloo, OnTARIO, CANADA N2L 3G1

E-mail address: slu@math.uwaterloo.ca

R.I.M.S., Kyoto University, Kyoto, JaPAN 606

E-mail address: miyaoka@hakobe.kurims.kyoto-u.ac.jp 